\title{
Dirofilariose canina na llha de São Luís, Nordeste do Brasil: uma zoonose potencial
}

\author{
Canine heartworm on São Luís Island, \\ Northeastern Brazil: a potential zoonosis
}

Silvia Maria Mendes Ahid 1

Ricardo Lourenço-de-Oliveira 2

Lauro Queiroz Saraiva 3

\footnotetext{
1 Departamento de Patologia, Escola de Medicina Veterinária, Universidade Estadual do Maranhão. Campus Paulo VI, Tirirical, São Luís, MA

65000-000, Brasil.

2 Departamento de

Entomologia, Laboratório

de Transmissores de

Haemoprotozoários,

Instituto Oswaldo Cruz.

Av. Brasil 4365,

Rio de Janeiro, RJ

21045-900, Brasil.

3 Secretaria Municipal

de Abastecimento.

Rua 35, quadra 34, casa 17 Jardim São Cristóvão,

São Luís, MA,

65000-000, Brasil.
}

Abstract A survey on the prevalence of canine heartworm was conducted in 1,495 dogs from Maranhão Island, State of Maranhão, Northeastern Brazil, from 1991 to 1994, by testing for microfilariae in blood. Of the total, 1,358 (12.8\% of which were infected) were dogs with no known history; they included 1,265 homel ess animals ( $10.3 \%$ with microfilariae) and 93 kept by owners at the time the survey was conducted ( $37.8 \%$ of which were infected). Prevalence is high among dogs captured and/or living al ong the seashore. Examples of such high coastal preval ence rates were found in Olho d'Água and Calhau ( $46 \%$ and 43\%, respectively). The search for mi crofilariae in blood samples of 137 exclusively domiciliary dogs with a known history showed that $43 \%$ of these animals were infected, confirming transmission of heartworm on the island. This was the first survey formally published on canine dirofilariasis in Northeastern Brazil. Since D. immitis is infective to man and prevalence of the parasite is high, particularly along the coast of Maranhão Island, human cases of dirofilariasis may be expected. Local physicians should consider this parasi te among the possible causes of solitary lesions in humans living in this area.

Key words Dirofilariasis; Dirofilaria sp.; Zoonosis

Resumo Um inquérito sobrea prevalência da di rofilariose canina foi realizado entre 1.495 cães de 64 localidades na Ilha de São Luís, de 1991 a 1994, através da pesquisa de microfilárias. Desses, 1.358 cães ( $12,8 \%$ positivos) não tinham história pregressa conhecida, sendo 1.265 errantes (10,3\% microfilarêmicos) e 93 domiciliados (37,8\%). A dirofilariose foi detectada em 46 das localidade, porém a prevalência da infecção aumenta consideravelmente quando se trata de cães da orla marítima, de onde procederam $47 \%$ dos an imais positivos. Em al guns bairros costeiros, a prevalência é el evada, como em Olho d'Água e Calhau (46\% e 43\% positivos, respectivamente). A pesquisa de microfilárias em 137 cães estritamente domi ciliados (DO) e com história pregressa conheci da (43\% mi crofilarêmicos) permitiu a comprovação da existência de transmi ssão na Il ha. Este estudo é o primei ro dessa natureza e abrangência realizado no Nordeste do País. Sabendo-se quea dirofilariose é uma zoonose, e diante da el evada prevalência de cães microfilarêmi cos, particularmente os domiciliados da orla marítima da Il ha, chama-se a atenção para que se consi dere tal parasitose como possível causa de lesões pulmonares solitárias na população humana local e naquel as assentadas em áreas com clima e pai sagem semel hantes no Nordeste. Palavras-chave Dirofilariose; Dirofilaria sp.; Zoonose 


\section{Introdução}

Dentre as enfermidades classificadas como zoonoses, encontra-se a dirofilariose, causada por um nematóide filarídeo, a Dirofilaria immitis (Leidy, 1856), cuja transmissão se dá às custas de mosquitos Culicidae (Parker, 1993). Vários casos de dirofilariose pulmonar humana vêm sendo registrados no mundo, inclusive no Brasil (Dashiell, 1961; Robinson et al., 1977; OMS, 1979; Merril et al., 1980; Rodrigues-Silva et al., 1995; Caldas, 1996; Campos et al., 1997).

Os vermes alcançam a forma adulta no coração direito e artérias pulmonares do cão, gato e outros mamíferos (Acha \& Szyfres, 1986) e, ocasionalmente, podem ser encontrados fora do sistema vascular (Abbott, 1961; Spratt et al., 1971).

A D. immitis vem sendo encontrada desde o Sul da Europa à América do Sul, em regiões tropicais, subtropicais e em determinadas áreas temperadas (Knight, 1977; Guerrero et al., 1992). A dirofilariose canina é enzoótica em muitas áreas, ao passo que a infecção entre os felinos é muito menos comum, sendo o cão considerado a principal fonte de infecção para os mosquitos vetores (Labarthe et al., 1998a, b).

No Brasil, considerando-se a extensão territorial e os variados climas, a distribuição e a prevalência da dirofilariose canina é pouco conhecida. Guerrero et al. (1992) assinalaram uma prevalência de $9,2 \%$ para todo o País, porém os níveis da endemicidade variam. No litoral sul e sudeste, a prevalência varia desde $13,7 \%$ e $24,8 \%$ nas cidades do Rio de Janeiro e Niterói, respectivamente (Labarthe et al., 1997), até $61,53 \%$ em Guarujá, São Paulo (Larsson et al., 1987). No Nordeste, onde as informações sobre a prevalência da dirofilariose são restritas, obtidas de amostragens pequenas, às vezes viciadas, e comunicadas apenas em reuniões científicas, o percentual de cães infectados varia de $18,7 \%$ em áreas semi-áridas, como Teresina, Estado do Piauí (I. L. Mendonça, comunicação pessoal) ), a 43,7\% em São Luís, Maranhão (Ahid \& Saraiva, 1993).

A presente investigação trata da ocorrência da dirofilariose canina na Il ha de São Luís e discute a possibilidade da transmissão do parasito canino a humanos nas áreas mais atingidas. Consiste no primeiro inquérito dessa natureza e abrangência realizado na Região Nordeste do Brasil.

\section{Material e métodos}

Amostras de sangue de um total de 1.495 cães da Il ha de São Luís foram examinadas no período de 1991 a 1994. Desses, 1.358 compreendiam 1.265 cães apreendidos aleatoriamente nas ruas de 64 localidades da Ilha, pelo Centro de Controle de Zoonoses, da Secretaria Municipal de Saúde de São Luís, no período de janeiro de 1991 a dezembro de 1993. Compreendiam, ainda, 93 cães domiciliados (SO), levados espontaneamente por seus proprietários ao Hospital Veterinário da Universidade Estadual do Maranhão (HV), de maio a dezembro de 1994, à procura de tratamento para diferentes patologias. Porém, esses proprietários desconheciam ambos o local de nascimento e o tempo de permanência do animal na atual e em anteriores residências. Passamos a considerar o local onde os animais haviam sido apreendidos ou residiam no momento em que a pesquisa foi efetuada como a sua origem.

Adicional mente, 137 cães estritamente domiciliados (DO) foram examinados no próprio domicílio, em 1994, levando-se em consideração toda a sua história pregressa, registrandose informações, tais como: raça, sexo, idade, local de nascimento e tempo de residência, a fim de permitir a identificação da real autoctonia dos casos de acordo com os bairros e localidades da llha.

Em todas as amostragens, foram incluídos somente cães com idade igual ou superior a 11 meses. Consideraram-se as características da localidade de origem, quanto à proximidade com a orla marítima, à presença da vegetação de mangue e ao tipo de agrupamento, se urbano ou rural.

A Il ha ( $2045^{\prime} \mathrm{S} 44 \circ 20^{\prime} \mathrm{W}$ e $2025^{\prime} \mathrm{S} 44 \circ 01^{\prime} \mathrm{W}$ ) possui uma área em torno de $914 \mathrm{~km}^{2}$, com altitudes variando de $0 \mathrm{~m}$ a $200 \mathrm{~m}$, e está localizada às margens das Baías de São Marcos e São José, no Estado do Maranhão, Nordeste do Brasil (Figura 1). O clima é do tipo tropical da zona equatorial, isto é, quente e semi-úmido, com quatro a cinco meses secos. A temperatura média anual acha-se em torno de $26{ }^{\circ} \mathrm{C}$, a umidade relativa média oscila entre $70 \%$ e $80 \%$ e o índice anual pluviométrico é de $2.340 \mathrm{~mm}$. Boa parte da Il ha sofreu alteração antrópica, mas preservam-se áreas com vegetação nativa, como a herbácea e arbórea típica de restinga, distribuída nos locais de influência marinha, e os mangues, que se acham instalados particularmente nos ambiente salobros dos estuários dos rios.

Fez-se punção da veia safena de cada animal, coletando-se $3 \mathrm{ml}$ de sangue em frasco es- 
Figura 1

Localização das áreas onde foram coletadas as amostras de sangue de cães para a pesquisa de microfilárias de Dirofilaria immitis, na Ilha de São Luís, Estado do Maranhão, de 1991 a 1994. 1 - O rla marítima. 2 - Áreas urbanas com mangue nas proximidades. 3 - Áreas urbanas sem mangue nas proximidades. 4 - Áreas semi-rurais e sem mangue nas proximidades. 5 - Áreas rurais.

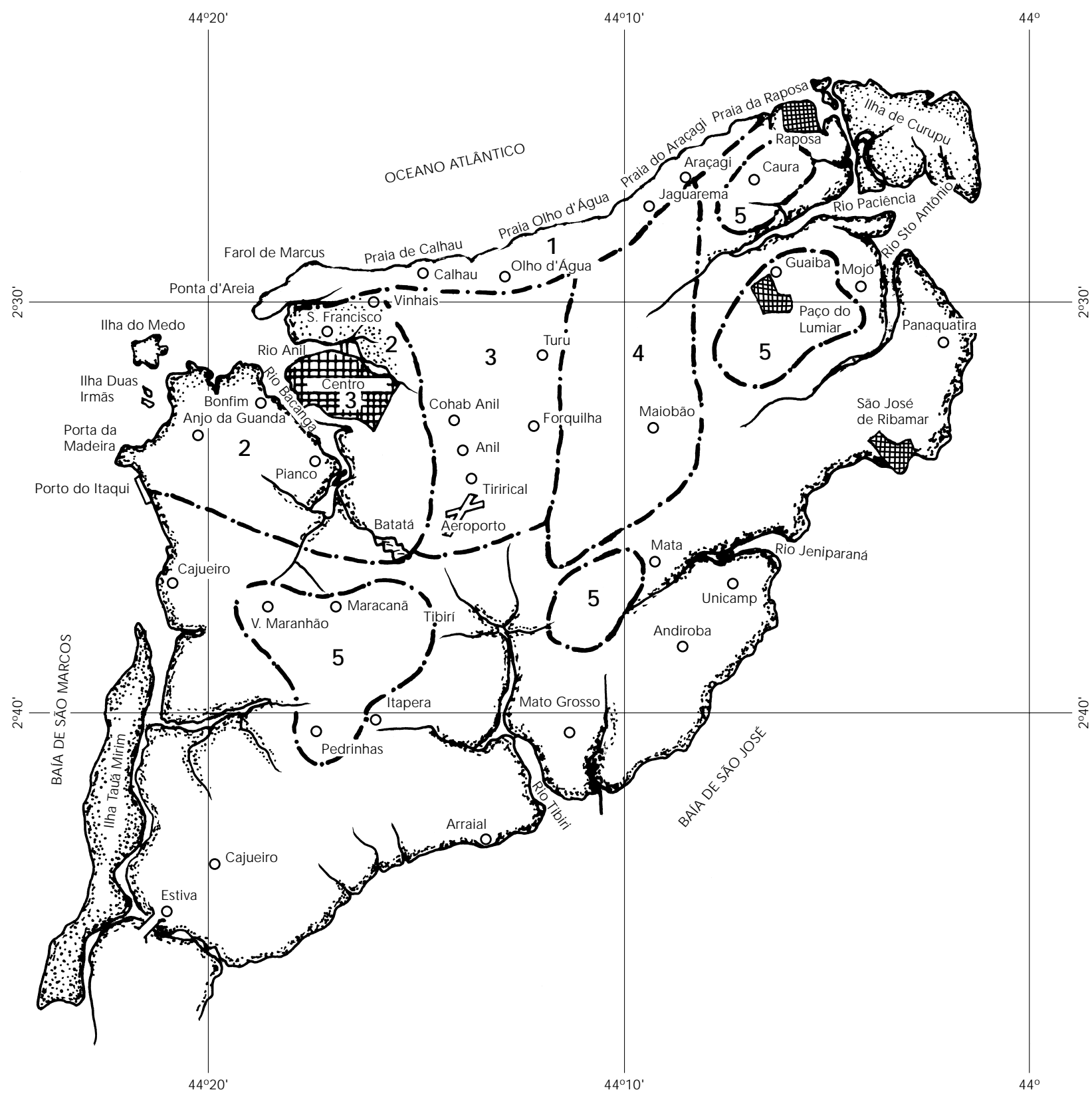

Centros urbanos

Mangue

ind

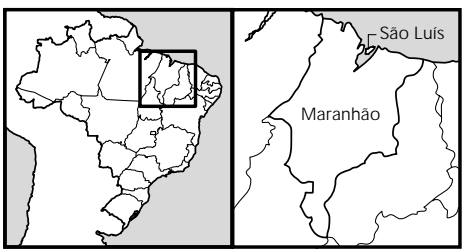


téril contendo 0,03 ml de EDTA (sal sódico de ácido etileno diamino tetracético a 10\%). A pesquisa das microfilárias de D. immitisfoi feita utilizando-se a técnica de Knott (1939), modificada por Newton \& Wright (1956).

Necropsiaram-se doze cães, de ambos os sexos, de idade variada, apresentando microfilaremia, com os objetivos de se verificar a freqüência dos vermes adultos e de se confirmar a etiologia das infecções locais. Cada órgão era examinado individualmente, anotando-se a infrapopulação encontrada. Os helmintos detectados eram mortos com AFA (álcool - formol ácido acético) aquecido, permanecendo nesta solução por 24 horas, sendo subseqüentemente transferidos para álcool a 70\% glicerinado (10\%) até a sua identificação.

Para análise estatística, estabeleceu-se a aleatoriedade quanto ao sexo, raça, origem e idade de todos os animais envolvidos na investigação. Usou-se o teste de Mantel-Haenszel na análise das variáveis, com limite de confiança de $95 \%$.

\section{Resultados e discussão}

Microfilárias de D. immitis foram detectadas em 224 dos 1.495 cães examinados, aproximadamente $15 \%$. Neste total, porém, acham-se animais de diferentes categorias. No conjunto de 1.358 cães de procedência duvidosa, a prevalência de microfilárias de $D$. immitis foi de $12,2 \%$. Tomando-se separadamente as 1.265 amostras sangüíneas coletadas exclusivamente de cães errantes, verificou-se que 130 eram positivas (10,3\%), sendo $62,6 \%$ dessas amostras de machos, com prevalência de cerca de $10 \%$ para ambos os sexos ( Tabela 1). O percentual de cães positivos variou em torno de 8,8\% para as amostras coletadas em 1991, 15,6 \% para as de 1992 e $8 \%$ para as de 1993.
A prevalência da dirofilariose entre os 1.265 cães errantes da Il lha (10,3\%) é inferior às observadas por Ahid \& Almeida (1991) em inquérito preliminar na mesma região (24,4\%) e por Mendonça (I. L. Mendonça, comunicação pessoal), em Teresina (18,7\%), quando estudaram populações caninas similares. Tais diferenças talvez se devam à origem e ao tamanho da amostra, bem como à amplitude da área coberta. Não encontramos diferença estatisticamente significante quanto à prevalência da dirofilariose entre os sexos dos animais ( $p=0,7582)$, enquanto Mendonça (I. L. Mendonça, comunicação pessoal) observou prevalências distintas: $15,44 \%$ e 3,25\% entre machos e fêmeas errantes, respectivamente.

Por outro lado, quando considerados os cães de procedência também duvidosa, porém domiciliados (SO) no momento em que a pesquisa foi realizada, a prevalência foi bem maior que aquela dentre os exclusivamente errantes (10,3\%). Isto é, das 93 amostras SO coletadas, $35(37,8 \%)$ revelaram-se positivas para microfilárias da D. immitis, sendo 59 fêmeas (23 positivas - 39\%) e 46 machos (22 positivos - 48\%). A diferença nas prevalências supracitadas pode ser explicada pela própria amostragem, embora em ambos os casos tenha sido aleatória. Com efeito, observou-se que os animais levados à procura de serviço veterinário no HV originavam-se, geralmente, de apenas 15 bairros, seis dos quais localizados na orla marítima, enquanto os cães vadios foram buscados nas mais variadas e remotas áreas da Ilha.

Na Tabela 2, apresentamos a distribuição dos 1.358 cães examinados e o percentual de positividade para microfilárias de $\mathrm{D}$. immitis de acordo com a sua origem. Nota-se que cães capturados em 46 das 64 localidades pesquisadas foram positivos. Agrupando-se as localidades quanto a algumas características, tais como aspecto de cobertura vegetal dominante e

Tabela 1

Prevalência de microfilárias de Dirofilaria immitis em 1.265 cães errantes capturados em 64 localidades da Ilha de São Luís, Estado do Maranhão, Brasil, de 1991 a 1993, de acordo o sexo. Número de cães examinados (n) e portadores de microfilárias (Mf).

\begin{tabular}{|c|c|c|c|c|c|c|c|c|}
\hline \multirow[t]{2}{*}{ Sexo } & \multicolumn{2}{|c|}{1991} & \multicolumn{2}{|c|}{1992} & \multicolumn{2}{|c|}{1993} & \multicolumn{2}{|c|}{ Total } \\
\hline & $\mathrm{Mf} / \mathrm{n}$ & $\%$ & $\mathrm{Mf} / \mathrm{n}$ & $\%$ & $\mathrm{Mf} / \mathrm{n}$ & $\%$ & $\mathrm{Mf} / \mathrm{n}$ & $\%$ \\
\hline Fêmeas & $11 / 192$ & 6 & $27 / 90$ & 30 & 9/191 & 5 & $47 / 473$ & 10,0 \\
\hline Machos & $37 / 352$ & 10,5 & $20 / 211$ & 9,5 & $26 / 229$ & 11 & $83 / 792$ & 10,5 \\
\hline Total & $48 / 544$ & 8,8 & $47 / 301$ & 15,6 & $35 / 420$ & 8 & $130 / 1.265$ & $10,3^{*}$ \\
\hline
\end{tabular}

* Limite de confiança 95\% (8,7\%-12,1\%); Mantel-Haenszel: Qui-quadrado =0,09 ( $p=0,75828491)$. 
nível de urbanização, observa-se que a maior prevalência de amostras positivas se deu nos bairros da orla marítima (24\%), dentre os quais destacam-se os bairros de Olho d'Água (46\%), Alto do Cal hau (43\%) e Calhau (25\%). Todavia, percentuais de positividade acima de $10 \%$ também foram observados em outras localidades, com características tanto rurais, quanto urbanas, sendo a mais baixa prevalência (7\%) detectada em localidades da zona rural. De fato, $47 \%$ do total de cães positivos foram procedentes de bairros da orla marítima, ao passo que a área rural concorreu com apenas 5\%. Embora esses dados sugiram haver maior concentração de cães positivos nos bairros costeiros, a história pregressa dos animais considerados acima era pouco conhecida. Ou seja, a provável mobilidade desses cães entre os bai rros não nos permitia concluir se os mesmos teriam adquirido a infecção pela D. immitis nos locais por onde vagavam ou em que residiam quando foram apreendidos e/ ou trazidos para exame no HV.

Com o propósito de se confirmar tanto a autoctonia, quanto a elevada prevalência da dirofilariose na orla marítima, buscou-se coletar amostras sangüíneas de 137 cães domiciliados (DO). A infecção foi diagnosticada em 59 desses cães (43\%). Dos animais examinados, $43(31 \%)$ residiam no Calhau, quarenta (29\%), no Olho d'Água, e 54 (40\%) distribuíam-se entre sete outras localidades, desde a orla marítima até a zona rural (Tabela 3). As prevalências maiores foram observadas dentre os animais do Olho d'Água, onde 52,5\% dos cães autóctones achavam-se infectados, e dentre aqueles das raças Fila - dos seis animais examinados, seis estavam positivos (6/6); Weimarand (3/3); Rotweiller (4/5 - 80\%); Dog (5/ 7 - 71\%); Coker e Pastor (em ambas 5/10 - 50\%). Dentre os animais sem raça definida (SRD), a prevalência foi de $48 \%$ (14/29), ao passo que os das raças Doberman (10/34 - 29\%) e Akita (2/7 - 29\%) apresentaram as menores prevalências.

Ainda quanto aos cães domiciliados, sejam SO ou DO, viu-se que a prevalência da infecção entre machos ( $48 \%$ e $47 \%$, respectivamente) é apenas ligei ramente maior que em fêmeas ( $39 \%$ e $40 \%$, respectivamente). Contudo, alguns autores sugerem que cães machos sofrem maior grau de exposição à infecção ou mesmo que são mais susceptíveis ao parasitismo pelos filarídeos que as fêmeas (Thrasher et al., 1968; Pennington \& Phelps, 1969; Prouty, 1972; Alves et al., 1993).

A prevalência de cães com microfilárias de D. immitis aumenta progressivamente com a faixa etária, como já se tem verificado em várias partes do mundo (Beugnet et al., 1994).
Tabela 2

Procedência de 1.358 cães examinados, sendo 1.265 errantes e 93 domiciliados, porém sem história pregressa conhecida, de acordo com 64 localidades da llha de São Luís, Estado do Maranhão, Brasil, de 1991 a 1994. Número de cães examinados $(n)$, portadores de microfilárias $(M f)$ e freqüência no total de animais positivos (MF+).

\begin{tabular}{lrrrc}
\hline Localidades & $\mathrm{n}$ & $\mathrm{Mf}$ & $\%$ & $\%(\mathrm{MF}+)$ \\
\hline Orla marítima & & & & \\
$\quad$ Alto do Calhau & 14 & 6 & 4 & 43 \\
Calhau & 67 & 17 & 10 & 25 \\
Cohafuma & 26 & 4 & 2 & 15 \\
Cohajap & 28 & 3 & 2 & 11 \\
Divinéia & 46 & 6 & 4 & 13 \\
Olho d'Água & 68 & 31 & 19 & 46 \\
Ponta da Areia & 21 & 3 & 2 & 14 \\
Planalto Vinhais & 15 & 4 & 2 & 27 \\
Shaloon & 42 & 4 & 2 & 9,5 \\
Subtotal & 327 & 78 & 47 & 24 \\
\hline
\end{tabular}

\begin{tabular}{lrrrr}
$\begin{array}{l}\text { Área urbana com mangue } \\
\text { nas proximidades }\end{array}$ & & & & \\
Anjo da Guarda & 40 & 2 & 1 & 5 \\
Bom J esus & 18 & 1 & 1 & 6 \\
Coroado & 57 & 4 & 2 & 7 \\
Coroadinho & 48 & 3 & 2 & 6 \\
Filipinho & 14 & 2 & 1 & 14 \\
Ivar Saldanha & 12 & 1 & 1 & 8 \\
Lira & 17 & 1 & 1 & 6 \\
Madre Deus & 11 & 1 & 1 & 9 \\
Recanto Vinhais & 6 & 3 & 2 & 50 \\
Redenção & 8 & 1 & 1 & 12,5 \\
Renascença & 2 & 0 & 0 & 0 \\
Sá Viana & 21 & 2 & 1 & 9,5 \\
São Francisco & 21 & 3 & 2 & 14 \\
Sitio Leal & 19 & 1 & 1 & 5 \\
Subtotal & 294 & 25 & 15 & 8,5 \\
\hline
\end{tabular}

Área urbana sem vegetação

de mangue

$\begin{array}{lllll}\text { Alemanha } & 21 & 1 & 1 & 5\end{array}$

Angelim

Anil

Atenas

Aurora

Bairro Fátima

Bequimão

Centro

Cohab

Cohatrac

Fé em Deus

Itapiracó

J oão Paulo

J oão de Deus

Liberdade

Planalto Anil

21
24
1
15
31
37
12
25
28
2
31
63
57
28
1

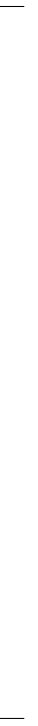
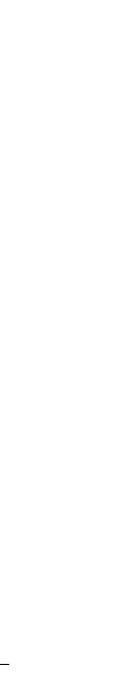
Tabela 2 (cont.)

Procedência de 1.358 cães examinados, sendo 1.265 errantes e 93 domiciliados, porém sem história pregressa conhecida, de acordo com 64 localidades da Ilha de São Luís, Estado do Maranhão, Brasil, de 1991 a 1994. Número de cães examinados (n), portadores de microfilárias (Mf) e freqüência no total de animais positivos $(M F+)$

\begin{tabular}{|c|c|c|c|c|}
\hline Localidades & $\mathrm{n}$ & $M f$ & $\%$ & $\%(M F+)$ \\
\hline \multicolumn{5}{|c|}{$\begin{array}{l}\text { Área urbana sem vegetação } \\
\text { de mangue (cont.) }\end{array}$} \\
\hline Santos Dumond & 1 & 0 & 0 & 0 \\
\hline Turu & 40 & 4 & 2 & 10 \\
\hline Vinhais & 13 & 1 & 1 & 8 \\
\hline Vila Palmeira & 14 & 1 & 1 & 7 \\
\hline Subtotal & 465 & 37 & 22 & 8 \\
\hline \multicolumn{5}{|l|}{ Área semi-rural } \\
\hline Alameda Açailândia & 3 & 0 & 0 & 0 \\
\hline Bom Milagre & 1 & 0 & 0 & 0 \\
\hline Cidade Operária & 112 & 15 & 9 & 13 \\
\hline Maiobão & 2 & 0 & 0 & 0 \\
\hline Pirapora & 16 & 1 & 1 & 6 \\
\hline Pavão Filho & 1 & 0 & 0 & 0 \\
\hline Subtotal & 135 & 16 & 10 & 12 \\
\hline \multicolumn{5}{|l|}{ Área rural } \\
\hline Codozinho & 10 & 0 & 0 & 0 \\
\hline Inhaúma & 2 & 0 & 0 & 0 \\
\hline J ardim Tropical & 1 & 0 & 0 & 0 \\
\hline Maiobinha & 2 & 0 & 0 & 0 \\
\hline Paço do Lumiar & 1 & 0 & 0 & 0 \\
\hline São Raimundo & 1 & 0 & 0 & 0 \\
\hline Santa Clara & 1 & 0 & 0 & 0 \\
\hline Tibirí & 9 & 1 & 1 & 11 \\
\hline Vila Brasil & 10 & 1 & 1 & 10 \\
\hline Vila Flamengo & 10 & 1 & 1 & 10 \\
\hline Vila Sarney & 20 & 2 & 1 & 10 \\
\hline Vila Riod & 11 & 1 & 1 & 9 \\
\hline Vila J anaina & 24 & 2 & 1 & 8 \\
\hline Vila Maranhão & 30 & 1 & 1 & 3 \\
\hline Vila São J osé & 5 & 0 & 0 & 0 \\
\hline Subtotal & 137 & 9 & 5,5 & 7 \\
\hline Total & 1.358 & 165 & 100 & 12,2 \\
\hline
\end{tabular}

Na Ilha de São Luís, a prevalência variou de $44,3 \%$ entre os animais de um a três anos a $100 \%$ dentre os acima de nove anos (Tabela 4). Nenhuma microfilária foi detectada em cães com menos de um ano de idade. Os animais com idade entre dois a seis anos compreenderam $86,4 \%$ dos casos positivos. Em Recife, Alves et al. (1993) encontraram índice de 76,92\% de positividade entre os cães com idade superior a um ano.

O resultado do exame dos cães domiciliados mostrou perfil semelhante àquele observado entre os vadios, confirmando a elevada prevalência da dirofilariose nos bai rros costeiros. Com isso, conclui-se que, na llha de São Luís, a prevalência da dirofilariose aumenta à medida que se aproxima da orla marítima, seja para cães vadios, ou domiciliados, ao passo que, nas demais áreas estudadas, a proporção de positivos é bem mais baixa, como nas localidades rurais e semi-rurais, afastadas do oceano $(5 \% \mathrm{e}$ $9,6 \%$, respectivamente). Acrescente-se que, na orla marítima, a maior parte dos animais infectados é procedente de alguns bairros em particular, como Olho d'Água, não importando se o animal era errante $(18,8 \%$ do total de infectados) (Tabela 2) ou estritamente domiciliado $(52,5 \%)$ (Tabela 3). Esses bairros, curiosamente, são de classe média alta, com edificações geralmente de um ou dois pavimentos, onde ainda se preservam áreas de cobertura vegetal antrópica.

Sendo assim, confirmou-se que a orla marítima é um foco ativo de transmissão da D. immitis, mas é provável que a veiculação do parasito se dê em outras áreas da Ilha, já que cães infectados, porém não confirmados como autóctones, foram detectados em 46 de suas localidades.

Dos 12 cães positivos para microfilária e submetidos à necropsia, cinco alojavam vermes adultos de $D$. immitis somente no ventrículo direito (VD) (41,7\%); dois no VD e na artéria pulmonar (AP) $(16,7 \%)$; dois no tronco arterial pulmonar (TAP) (16,7\%); um no VD e válvula ventricular esquerda; um simultaneamente no VD, AP, TAP, e outro al ojando no VD, AP, TAP, átrio direito e tecido subcutâneo torácico direito $(8,3 \%$, cada). Em quatro cães (33,3\%) foram encontradas tumorações esofagianas contendo Spirocerca lupi Rudolphi.

Computando-se em conjunto os 230 cães domiciliados examinados no presente estudo, respectivamente $93 \mathrm{SO}$ e $137 \mathrm{DO}$, verificamos que 94 deles (40,8\%) achavam-se com microfilárias de D. immitis circulantes. Este percentual é bastante superior ao observado em cães domiciliados de outras áreas do Brasil, como o 
Tabela 3

Prevalência de microfilárias de D. immitis em 137 cães autóctones e estritamente domiciliados (DO) de 15 localidades da Ilha de São Luís, Estado do Maranhão, Brasil, examinados em 1994. Número de cães examinados (n) e portadores de microfilárias (Mf).

\begin{tabular}{|c|c|c|c|c|c|}
\hline \multirow[t]{2}{*}{ Local } & \multicolumn{2}{|c|}{ Examinados } & \multicolumn{3}{|c|}{ Positivos para dirofilária } \\
\hline & $\mathrm{n}$ & Freq. (\%) & $M f$ & $\mathrm{Mf} / \mathrm{n}(\%)$ & Freq \\
\hline Alto Calhau & 17 & 12 & 5 & 29 & 8,5 \\
\hline Anil & 1 & 1 & 0 & 0 & 0 \\
\hline Calhau & 43 & 31 & 22 & 51 & 37 \\
\hline Cohafuma & 8 & 6 & 2 & 25 & 3 \\
\hline Olho d'Água & 40 & 29 & 21 & 52,5 & 36 \\
\hline Planalto Vinhais & 20 & 15 & 7 & 35 & 12 \\
\hline Renascença & 2 & 1,5 & 0 & 0 & 0 \\
\hline São Francisco & 5 & 4 & 2 & 40 & 3 \\
\hline Turu & 1 & 1 & 0 & 0 & 0 \\
\hline Total & 137 & 100 & 59 & 43 & 100 \\
\hline
\end{tabular}

Qui-Quadrado $=9,04 ; p=0,33933172$.

Tabela 4

Freqüência de microfilárias de Dirofilaria immitis, em relação à faixa etária, em 137 cães domiciliados (DO), de 15 localidades da Ilha de São Luís, Maranhão, Brasil, em 1994. Número de cães examinados (N) e portadores de microfilárias (Mf).

\begin{tabular}{|c|c|c|c|}
\hline \multirow[t]{2}{*}{ Classes de idades } & \multirow[t]{2}{*}{ Freqüência relativa (\%) } & \multicolumn{2}{|c|}{ Dirofilariose } \\
\hline & & $\mathrm{Mf} / \mathrm{N}$ & $\%$ \\
\hline ] $11 \mathrm{~m}-1 \mathrm{a}]$ & 17 & $0 / 23$ & 0 \\
\hline ] 1 - 3 ] & 44,5 & $27 / 61$ & 44,3 \\
\hline ] $3-6$ ] & 32 & $24 / 44$ & 54,5 \\
\hline ] $6-9$ ] & 3 & $3 / 4$ & 75 \\
\hline$>9$ anos & 4 & $5 / 5$ & 100 \\
\hline Total & 100 & $59 / 137$ & 43 \\
\hline
\end{tabular}

Qui-Quadrado $=28,07 ;$ valor de $p=0,00001206 ; m=$ meses; $a=$ ano.

Estado de São Paulo (8,8\%), segundo Larsson (1990), e Recife, em Pernambuco (26\%) (Alves et al., 1993).

A prevalência da dirofilariose entre os cães domiciliados na Il ha de São Luís foi aqui avaliada apenas com base na microfilaremia. Considerando-se que esta pode ser transitória ou mesmo ausente em infecções por D. immitis (Rawlings et al., 1982; Grieve et al., 1986), estima-se que a real prevalência entre esses animais deva ser bem mais alta que $40,8 \%$. Diante dessas cifras e do potencial zoonótico da dirofilariose, sugere-se que profissionais da área de saúde atentem para a possibilidade de D. immitis ser a causa de lesões pulmonares solitá- rias na população humana da Ilha de São Luís, especialmente naquelas assentadas junto à orla marítima. A elevada prevalência da infecção entre cães domiciliados em comparação com os animais errantes aponta para maior freqüência da transmissão do nematódeo nos ambientes domiciliar e peridomiciliar, o que aumenta a possibilidade do contato do homem com mosquitos infectados na Ilha de São Luís. 
Referências

ABBOTT, P. K., 1961. Dirofilaria immitis in the peritoneal cavity. Australian Veterinary Journal, 37:467.

ACHA, P. N. \& SZYFRES, B., 1986. Zoonosis y Enfermedades Transmisibles Comunes al Hombre y a los Animales. 2a Ed. Publicación Científica no. 503. Washington: Organización Panamericana de la Salud.

AHID, S. M. M. \& ALMEIDA, V. M., 1991. Identificação da dirofilariose canina em São Luís - MA. Revista do Instituto de Medicina Tropical deSão Paulo, 33 (Sup. 8):34.

AHID, S. M. M. \& SARAIVA, L. Q., 1993. Ocorrência da filariose canina no Município de São Luís - MA. Revista Brasileira de Parasitologia Veterinária, 2 (Sup. 1):70.

ALVES, L. C.; COLE, E. F. \& ATHAYDE, A. C. R., 1993. Prevalência da filariose canina no bairro de Dois Irmãos, Recife-PE. Revista Brasileira de Parasitologia Veterinária, 2 (Sup. 1):68.

BEUGNET, F.; ROUS, V.; LEUS, M. \& CHARDOMET, L., 1994. Effect of age in cardiopulmonary canine dirofilariasis. Choice of date for commencement of chemoprophylaxis. Revue de MedecineVetérinaire, 145:59-64.

CAM POS, J. R. M.; BARBAS, C. S. V.; FILOMENO, L. T. B.; FERNANDEZ, A.; MINAMOTO, H.; BARBAS FILHO, J. V. \& JATENE, F. B., 1997. Human pulmonary dirofilariasis. Analysis of 24 cases from São Paulo, Brazil. Chest, 112:729-733.

CALDAS, E. M., 1996. O médico veterinário na saúde pública. Revista do Consel ho Federal de Medicina Veterinária, 2:15-17.

DASHIELL, G. F., 1961. A case of dirofilariasis involving the lung. American Journal Tropical Medicine and Hygiene, 10:37-39.

GRIEVE, R. B.; GLICKM AN, L. T. \& BATER, A K., 1986. Canine Dirofilaria immitis infection in na hyperenzootic area: examination by parasitologic finds at necropsy and by two serological methods. American Journal Veterinary Research, 47:392-393.

GUERRERO, J.; DUCOS, H. J.; GENCHI, C.; ROJO, F.; GOMEZ-BAUTISTA, M. \& VARELA, M. C., 1992. Update on the distribuition of Dirofilaria immitis in dogs from Southern Europa and Latin America. Proceedings of the Heartworm Symposium'92, pp. 31-37, Batavia: American Hookworm Society.

KNIGHT, D. H., 1977. Heartworm heart disease. Advances in Veterinary Science, 21:107-147.

KNOTT, J., 1939. A method for making microfilarial surveys on dog blood. Transactions of the Royal Society Tropical Medicine and Hygiene, 33:191196.

LARSSON, M. H. M. A., 1990. Prevalence of Dirofilaria immitis microfilarias in dogs from the state of São Paulo. Brazilian Journal of Veterinary Research and Animal Science, 27:183-186.

LARSSON, M. H. M.; IWASAKI, M.; FERNANDES FILHO, A.; DERRICO, A. A.; OGASSAWARA, S. \& DUTRA, L. F., 1987. Contribuição do diagnóstico clínico da dirofilariose canina. Revista da Faculdade de Medicina Veterinária e Zootecnia da Universidade de São Paulo, 24:47-59.

LABARTHE, N.; ALMOSNY, N.; GUERRERO, J. \& DUQUE-ARAÚJO, A. M., 1997. Description of the occurrence of canine dirofilariasis in the state of Rio de Janeiro, Brazil. Memórias do Instituto Oswaldo Cruz, 92:47-51.

LABARTHE, N.; SERRÃO, M. L.; MELO, Y. F.; OLIVEIRA, S. J. \& LOURENÇO-DE-OLIVEIRA, R., 1998a. Mosquito frequency and feeding habits in na enzootic canine dirofilariasis area in Niterói, State of Rio de Janeiro, Brazil. Memórias do Instituto Oswaldo Cruz, 93:145-154.

LABARTHE, N.; SERRÃO, M. L.; MELO, Y. F.; OLIVEIRA, S. J. \& LOURENÇO-DE-OLIVEIRA, R., 1998b. Potential vectors of Dirofilaria immitis (Leidy, 1856) in Itacoatiara, oceanic region of Niterói Municipality, Rio de Janeiro, Brazil. Memórias do Instituto Oswaldo Cruz, 93: 425-432.

MERRILL, J. R.; OTIS, J.; LOGAN, W. D. \& DAVIS, M. B., 1980. The dog heartworm (Dirofilaria immitis) in man. An epidemic pending or in progress? JAMA, 243:1066-1068.

NEWTON, W. L. \& WRIGHT, W. H., 1956. The occurrence of a dog filariid other than Dirofilaria immitis in the United States. Journal Parasitology, 42:246-258.

OMS (Organización Mundial de la Salud), 1979. Zoonosis parasitarias. Informe Técnico, 637:105106.

PARKER, B. M., 1993. Variation of mosquito (Diptera: Culicidae) relative abundance and Dirofilaria immitis (Nematoda: Filarioidea) vector potential in coastal North Carolina. Journal of Medical Entomology, 30:436-442.

PENNINGTON, N. E. \& PHELPS, C. A., 1969. Canine filariasis on Okanawa Ryukyu Island. Journal of Medical Entomology, 6:59-67.

PROUTY, D. L., 1972. Canine heartworm disease in Southeastern Michigan. Journal of the American Veterinary Medical Association, 16:1675-1676.

RAWLINGS, C. A; DAVE, D. L. \& MCCALL, J. W., 1982. Four types of occult Dirofilaria immitis infection in dogs. Journal of the American Veterinary Medical Association, 180:1323-1326.

ROBINSON, N. B.; CHAVEZ, C. M. \& CONN, J. H., 1977. Pulmonary dirofilariasis in man: a case report and review of the literature. Journal of Thoracic and Cardiovascular Surgery, 74:403-408.

RODRIGUEZ-SILVA, R.; MOURA, H.; DREYER, G \& REY, L., 1995. Human pulmonary dirofilariasis: a review. Revista do Instituto de Medicina Tropical deSão Paulo, 37:523-530.

SPRATT, D. M.; MALLET, D.; DERRINGTON, P. C. \& THOMAS, C., 1971. Male Dirofilaria immitis from the eye of a dog. Australian Veterinary Journal, 47:124.

THRASHER, J. P.; GOULD, K. G. \& LYNCH, M. J., 1968. Filarial infections of dogs in Atlanta, Georgia. Journal of the American Veterinary Medical Association, 153:1059-1063. 